


\section{DEU'TSCHE AKADEMIE DER WISSENSCHAFTEN ZU BERLIN}

INSTITUT FÜR GRIECHISCH-ROM ISCHE ALTERTUMSKUNDE

BERLINER BYZANTINISTISCHE ARBEITEN

BAN D 2

1956

AKA DEMIE - VERLAG - BERLIN 
GERTRUD BOHLIG

\section{UNTERSUCHUNGEN \\ ZUM RHETORISCHEN SPRACHGEBRAUCH \\ DER BYZANTINER}

MIT BESONDERER BERÜCKSICHTIGUNG

DER SCHRIFTEN

DES MICHAEL PSELLOS

MIT EINEM GELEITWORT

VON FRANZ DÖLGER

1956

AKA D E M I - VERLAG - B ERLIN 
Herausgeber der Reihe:

Johannes Irmscher

Redaktion dieses Bandes:

Hans Ditten

Erachienen im Akademie-Verlag GmbH., Berlin W 8, MohrenstraBe 39 Lizenz-Nr. $202 \cdot 100 / 424 / 56$

Hersteilung: Satz und Druck Tribane Druckerel III Leipzig III/18/36

Bestell- und Verlagsnummer 2065/2

Printed in Germany 